\title{
Fall Risk and Related Factors in Systemic Lupus Erythematosus
}

\author{
Meltem ALKAN MELIKOĞLU, ${ }^{1}$ Fatih BAĞCIER ${ }^{2}$ \\ ${ }^{1}$ Department of Physical Medicine and Rehabilitation, Division of Rheumatology, Medical Faculty of Atatürk University, Erzurum, Turkey \\ ${ }^{2}$ Department of Physical Medicine and Rehabilitation, Medical Faculty of Atatürk University, Erzurum, Turkey
}

\begin{abstract}
Objectives: This study aims to determine the fall risk in systemic lupus erythematosus (SLE) patients with an objective computerized technique and to evaluate the potential related risk factors for falls in these patients.

Patients and methods: A total of 48 female patients (mean age $37.8 \pm 12.6$ years; range 18 to 65 years) with SLE and 30 female controls (mean age $39.1 \pm 15.7$ years; range 19 to 66 years) were included. Age, disease duration, anamnesis of falls, fear of falling, and drugs used were recorded. Disease activity (measured with SLE disease activity index Safety of Estrogens in Lupus Erythematosus National Assessment modification) and damage (measured with Systemic Lupus International Collaborating Clinics/American College of Rheumatology Damage Index) were evaluated. For the evaluation of fall efficacy, we used the Falls Efficacy Scale International. Fall risk analysis was performed by using a computerized posturography device.

Results: Seven patients (15\%) had anamnesis of falls during the last 12 months, whereas only one control (3\%) had this anamnesis ( $p=0.042$ ). Fear of falling was reported by $40 \%$ of the patients and $30 \%$ of the controls $(p=0.042)$. Falls Efficacy Scale International scores were found to be higher in patients than in controls ( $p=0.042$ ). With the computerized system used, higher fall risk results were recorded in patients than in controls $(59.3 \pm 29.5$ and $35.8 \pm 26.9$, respectively; $\mathrm{p}<0.001$ ). Low, moderate, and high fall risks were recorded as $31 \%, 15 \%$, and $54 \%$ in the patients, respectively, and as $53 \%$, $23 \%$, and $23 \%$ in the controls, respectively. Low, moderate and high fall risk distribution was also worse in patients than in the controls ( $p=0.030$ ). No significant correlation was found between fall risk and the other factors evaluated, except Falls Efficacy Scale International, which was significantly correlated with both fall risk assessment and fall risk category in the patients $(r=0.311, p=0.031)$.

Conclusion: Fall risk was found to be higher in SLE patients than in controls. The higher fall risk in these patients seems to be affected by the disease itself rather than its other characteristics. Future studies investigating the possible coexisting balance problems in SLE may contribute to the management of the disease.

Keywords: Balance; fall risk; systemic lupus erythematosus.
\end{abstract}

Systemic lupus erythematosus (SLE) is a multisystem disease that has a broad range of manifestations. Balance, as a complex task, may be affected in SLE and this may cause postural instability and fall risk. A fall can be defined as "an unexpected event in which participants come to rest on the ground, floor, or other lower level" according to the consensus guidelines. ${ }^{1}$ Its consequences are highly variable and may include loss of confidence, injury, and even death. ${ }^{2}$ Although its etiology seems to be multifactorial, which includes a complex interaction of intrinsic, behavioral, or environmental risk factors, age and related comorbidities are considered to be the most important risk factors for falls in the normal population. ${ }^{3,4}$ Rheumatic diseases were suggested as the second strongest independent risk factor for serious fall-related injuries in elderly females. ${ }^{5}$ In spite of its reasonable commonness and often serious consequences in patients with rheumatic diseases, falls are still underestimated and poorly researched in this population. In studies about falls in patients with several rheumatic diseases, there is a wide range in the incidence of reported falls, which may be due to inconsistency in the attainment of data regarding falls. Suggested potential fall risk factors were also conflicting as a result of several different methodologies used in 
previous studies. Moreover, most of the previous data about fall risk in rheumatic diseases were achieved from patients with rheumatoid arthritis (RA).

To the best of our knowledge, no investigations have been conducted about the fall risk in patients with SLE. Determining the fall risk and related risk factors in these patients may lead to the awareness and prevention of falls and contribute to the management of the patients. In this study, we aimed to determine the fall risk in SLE patients with an objective computerized technique and to evaluate the potential related risk factors for falls in these patients.

\section{PATIENTS AND METHODS}

This cross-sectional study, which was conducted at Medical Faculty of Atatürk University between January 2015 and July 2015, included a total of 48 female patients with SLE (mean age 37.8 12.6 years; range 18 to 65 years) who met the 1997 American College of Rheumatology Revised Classification Criteria for SLE and 30 female controls (mean age 39.1 15.7 years; range 19 to 66 years) with similar age and sex profiles. ${ }^{6}$ Patients who were not able to cooperate or tolerate the fall risk evaluation and patients who had undergone previous orthopedic surgery or who had any other diseases were excluded. The study protocol was approved by the Medical Faculty of Atatürk University Ethics Committee. A written informed consent was obtained from each subject. The study was conducted in accordance with the principles of the Declaration of Helsinki.

The characteristics of the participants, including age, disease duration, active clinical findings, fall history (during the last 12 months), fear of falling, and drugs usage were recorded. In addition, disease activity (measured with SLE disease activity index Safety of Estrogens in Lupus Erythematosus National Assessment modification) and damage (measured with System Lupus International Collaborating Clinics/ American College of Rheumatology Damage Index Systemic Lupus International Collaborative Clinics/American College of Rheumatology damage index) were evaluated in patients with SLE. ${ }^{7}$
For the evaluation of fall efficacy in patients with SLE, we used the Falls Efficacy Scale International (FES-I), which is a self-reported questionnaire. $^{8}$ This scale is a short, easy-toadminister tool that measures the level of concern about falling during social and physical activities inside and outside the home, whether or not the person actually does the activity. It contains 16 items scored on a four-point Likert scale ( $1=$ not at all concerned to $4=$ very concerned), providing a total score ranging from 16 (absence of concern) to 64 (extreme concern). The FES-I was developed in a collaborative effort with members of the Prevention of Falls Network Europe $e^{8}$ and it was translated into several languages as well as our language. ${ }^{9}$ Fear of falling of the participants was also recorded (based on their replies as yes or no).

Fall risk analysis of patients with SLE and the controls were performed by using the Tetrax Interactive Balance System (Sunlight Medical Ltd., Tel Aviv, Israel) with the same technique directed by the user's guide of the device. Tetrax static posturography device has a computer and software system, and all of the data obtained from the device was the result of the software. The device uses a system of force gauges in four plates on which subject stands, and uses wave signals to digitally translate the vertical forces on the plates into a computer. Before the task, the patients were instructed to place their feet side by side as indicated by the foot-shaped outlines on the platform, and not to speak or move during the task. Following the same technique, sequence, and directions for all subjects, measurements were made in eight different positions (each position takes about 40 seconds; (i) head straight, eyes open, on a hard ground; (ii) head straight, eyes closed, on a hard ground; (iii) head straight, eyes open, on a soft ground (sponge under feet); (iv) head straight, eyes closed, on a soft ground; (v) head turned to the right, eyes closed, on a hard ground; (vi) head turned to the left, eyes closed, on a hard ground; (vii) neck fully extended, eyes closed, on a hard ground; and (viii) neck fully flexed, eyes closed, on a hard ground. ${ }^{10} \mathrm{~A}$ fall index derived from the Tetrax balance parameters has been developed to produce a score that will express the patient's risk of falling based on the specific balance factors that affect falling. Fall risk is a numeric value ranging from 0 to 100 , with three risk groups, indicating low (0 to 35), 
Table 1. Comparisons of factors between systemic lupus erythematosus patients and controls

\begin{tabular}{|c|c|c|c|c|c|c|c|c|c|c|c|}
\hline & \multicolumn{5}{|c|}{ Patients $(n=48)$} & \multicolumn{5}{|c|}{ Controls $(n=30)$} & \multirow[b]{2}{*}{$p$} \\
\hline & $\mathrm{n}$ & $\%$ & Mean \pm SD & Median & Min-Max & $\mathrm{n}$ & $\%$ & Mean \pm SD & Median & Min-Max & \\
\hline Age (year) & & & $37.8 \pm 12.6$ & & & & & $39.1 \pm 15.7$ & & & 0.200 \\
\hline BMI $\left(\mathrm{kg} / \mathrm{m}^{2}\right)$ & & & $33.5 \pm 5.7$ & & & & & $32.1 \pm 6.8$ & & & 0.310 \\
\hline Symptom duration (months) & & & $48.2 \pm 11.0$ & & & & - & & & & \\
\hline Falls history & & 15 & & & & & 3 & & & & 0.042 \\
\hline Fear of falling & & 40 & & & & & 30 & & & & 0.042 \\
\hline FES-I median & & & & 24 & $10-82$ & & & & 15 & $10-81$ & 0.042 \\
\hline Fall risk assessment $(0-100)$ & & & $59.3 \pm 29.5$ & & & & & $35.8 \pm 26.9$ & & & 0.001 \\
\hline \multicolumn{12}{|l|}{ Fall risk category } \\
\hline Low & 31 & & & & & 53 & & & & & \\
\hline Moderate & 15 & & & & & 23 & & & & & \\
\hline High & 54 & & & & & 23 & & & & & \\
\hline
\end{tabular}

moderate (36 to 57 ), or high (58 to 100$)$ risk of fall. ${ }^{11}$

\section{Statistical analysis}

All analyses were performed using the IBM SPSS version 20.0 software package (IBM Corp., Armonk, NY, USA). All of the data were examined for normality. Normally distributed data were expressed as the mean with standard deviation, otherwise as the median with ranges. Unpaired samples t test, Mann-Whitney U test, Chi-square and Spearman correlation tests were used for statistical analysis. $P$ values less than 0.05 were considered to be statistically significant.

\section{RESULTS}

The demographic features of the participants were summarized in Table 1. Fatigue (70\%), locomotor system involvement (60\%), skin findings (60\%), renal involvement (30\%), and hematological findings (20\%) were recorded in SLE patients. Patients were under the treatment of nonsteroidal anti-inflammatory drugs $(\mathrm{n}=2)$, steroids alone $(\mathrm{n}=1)$, hydroxychloroquine alone $(\mathrm{n}=11)$, hydroxychloroquine plus steroids $(\mathrm{n}=24)$, hydroxychloroquine, steroids, and azathioprine $(\mathrm{n}=8)$, and other drugs including cyclosporine-A and methotrexate $(\mathrm{n}=2)$.

Seven patients with SLE had anamnesis of falls during the last 12 months, whereas only one control had this anamnesis $(p=0.042)$. A total of $40 \%$ of the patients and $30 \%$ of the controls reported fear of falling $(p=0.042)$. FES-I scores were found to be higher in patients than in the controls ( $\mathrm{p}=0.042)$. With the computerized system used, higher fall risk results were recorded in the patients than in the controls $(p<0.001)$. Low, moderate, and high fall risks were recorded as $31 \%, 15 \%$, and $54 \%$ in the patients, respectively, and $53 \%, 23 \%$, and $23 \%$ in the controls, respectively. This distribution of low, moderate and high fall risk was also significantly worse in the patients than in the controls $(p<0.030)$. No significant correlation was found between fall risk and the other factors evaluated, except FES-I, which was significantly correlated with both fall risk assessment and fall risk category in patients $(\mathrm{r}=0.311, \mathrm{p}=0.031)$. The comparisons of the demographic features, anamnesis of falls, fear of falling, scores of FES-I, and fall risk assessments were shown in Table 1.

\section{DISCUSSION}

In this study, fall risk was found to be higher in SLE patients than in the controls. The higher fall risk in these patients seems to be affected by the disease itself rather than its other characteristics.

Falls, which may lead to important consequences, represent an important burden to health care resources worldwide. ${ }^{2}$ It is suggested that having several rheumatic diseases is the second strongest independent risk factor for serious falls, ${ }^{5}$ putting these patients at a greater risk of falling. Therefore, the definition of fall risk and its related factors in rheumatic diseases may contribute to its prevention and the management of these patients. In our study of evaluating fall risk and its related factors in patients with SLE 
by using an objective computerized technique, it has been demonstrated that fall risk was higher in patients with SLE than in healthy people, regardless of the patient's characteristics.

Although falls have been considered as a common event in patients with rheumatic diseases, fall risk research was limited in this population. In studies of falls in patients with several rheumatic diseases, there is a wide range in the reported incidence of falls, which may be due to inconsistency in the attainment of data regarding falls. In this limited data, patients with RA seem to be the most frequently investigated group. In previous studies, the incidence of falls has been reported in a wide range (10 to 50\%) in patients with RA, depending on the methodology of evaluating falls. ${ }^{2}$ In a study evaluating postural stability in patients with fibromyalgia, increased postural instability and increased frequency of falls were demonstrated in patients. ${ }^{12}$ In this study, worse postural performance and fall risk found in fibromyalgia patients compared to controls were found to be related to the sleep quality in the last 24 hours and level of fatigue.

Suggested potential fall risk factors were also conflicting as a result of several different methodologies used in previous studies. However, the strongest correlation with falls was reported as RA patient-reported outcomes. ${ }^{13}$ In our study, we could not find any relationship between fall risk and the age of the patient or disease duration. In previous studies investigating the incidence and risk factors for falls in people with RA, age was assessed as a risk factor for falls, but no significant association was found between age and fall frequency. ${ }^{2,14}$ This is an important finding for rheumatic diseases since studies in healthy older adults consistently report an increase in fall risk with increasing age. ${ }^{3,4}$ Also, no relationship could be demonstrated between the disease duration and fall risk in RA patients in previous studies. ${ }^{15}$ It may be considered that, similar to RA, higher fall risk compared to controls in SLE seems to be independent from the patient's age and disease duration.

Although some previous studies in RA demonstrated a relationship between fall risk and disease activity or its components, the other diseases did not show such an association in these patients. ${ }^{15-17}$ As a clinical feature, fatigue was also reported to be an independent predictor of future falls. ${ }^{17}$ Similar to our study design, Aydog et al. $^{18}$ determined that dynamic balance was affected by functional status, but not RA disease activity. In our study, we did not find a correlation between fall risk assessment and clinical findings and disease activity or damage. Since, to our knowledge, this is the first study evaluating fall risk in SLE, these results need to be confirmed by new studies in this patient population.

Drug usage may be another factor which should be questioned in relation to falls. Although there are no previous data on the relationship between drugs and falls in patients with SLE, previous studies on RA have demonstrated that various drugs, such as glucocorticoids (dose-dependent), diuretics, antihypertensives, antidepressants, and sedatives, can increase the risk of falling. ${ }^{2,14-16}$ However, in another study, steroid intake did not influence the fall risk assessment results in patients with RA. ${ }^{13}$ Since the previous data was based on a different disease, namely RA, different drugs, and different fall risk assessments, it may be difficult to consider these results together with our study. However, it seems to be reasonable that drugs may be considered as one of the possible factors related to fall risk in these patients. Further studies may clarify the association between drugs and fall risk in patients with SLE.

In our study, we found higher rates of fall anamnesis during the last 12 months and fear of falling in SLE patients compared to the controls. However, there was no association between these parameters and objectively obtained fall risk scores in patients. In previous studies on falls in RA patients, history of falls was reported to be an independent predictor of future falls. ${ }^{17}$ This may be due to different patient populations and different methodology used to evaluate fall risks. Also, in accordance with our data, two previous studies reported no association between fear of falling and falls. ${ }^{19,20}$

Falls present a frequent and substantial health problem among patients with rheumatic diseases. However, the investigation of falls and related factors can be problematic in studies. A definition of the term "fall" is frequently missing from falls research. ${ }^{21}$ The differences in fall rates in previous studies have been attributed to variation in the definition of a fall event. ${ }^{2}$ Also, most of the 
previous studies used "self-reported" falls data. Being the first study on falls in patients with SLE with an objective technique may increase the importance of our study.

A limitation of this study is its small sample size. Thus, a larger sample size including several involvements seen in SLE may provide more significant results.

In conclusion, by using a computerized technique, fall risk was found to be higher in SLE patients than in the controls in our study. The higher fall risk in these patients seems to be affected by the disease itself rather than its other characteristics. Future studies investigating the possible coexisting balance problems in SLE may contribute to the management of disease.

\section{Declaration of conflicting interests}

The authors declared no conflicts of interest with respect to the authorship and/or publication of this article.

\section{Funding}

The authors received no financial support for the research and/or authorship of this article.

\section{REFERENCES}

1. Lamb SE, Jørstad-Stein EC, Hauer K, Becker C. Development of a common outcome data set for fall injury prevention trials: the Prevention of Falls Network Europe consensus. J Am Geriatr Soc 2005;53:1618-22.

2. Brenton-Rule A, Dalbeth N, Bassett S, Menz HB, Rome $\mathrm{K}$. The incidence and risk factors for falls in adults with rheumatoid arthritis: a systematic review. Semin Arthritis Rheum 2015;44:389-98.

3. McKay C, Anderson KE. How to manage falls in community dwelling older adults: a review of the evidence. Postgrad Med J 2010;86:299-306.

4. Deandrea S, Lucenteforte E, Bravi F, Foschi R, La Vecchia C, Negri E. Risk factors for falls in community-dwelling older people: a systematic review and meta-analysis. Epidemiology 2010;21:658-68.

5. Bergland A, Wyller TB. Risk factors for serious fall related injury in elderly women living at home. Inj Prev 2004;10:308-13.

6. Hochberg MC. Updating the American College of Rheumatology revised criteria for the classification of systemic lupus erythematosus. Arthritis Rheum 1997;40:1725.

7. Gordon C, Bertsias G, Ioannidis JP, Boletis J, Bombardieri S, Cervera R, et al. EULAR points to consider for conducting clinical trials in systemic lupus erythematosus. Ann Rheum Dis 2009;68:470-6.

8. Yardley L, Beyer N, Hauer K, Kempen G, Piot-Ziegler C, Todd C. Development and initial validation of the Falls Efficacy Scale-International (FES-I). Age Ageing 2005;34:614-9.

9. Ulus Y, Durmus D, Akyol Y, Terzi Y, Bilgici A, Kuru O. Reliability and validity of the Turkish version of the Falls Efficacy Scale International (FES-I) in community-dwelling older persons. Arch Gerontol Geriatr 2012;54:429-33.

10. Akkaya N, Akkaya S, Atalay NS, Acar M, Catalbas $\mathrm{N}$, Sahin F. Assessment of the relationship between postural stability and sleep quality in patients with fibromyalgia. Clin Rheumatol 2013;32:325-31.

11. Ozdemir O, Gokce Kutsal Y. Fall risk assessment of elderly by using posturography. Turk J Geriatr 2009; $12: 177-80$.

12. Jones $\mathrm{KD}$, Horak $\mathrm{FB}$, Winters-Stone $\mathrm{K}$, Irvine JM, Bennett RM. Fibromyalgia is associated with impaired balance and falls. J Clin Rheumatol 2009;15:16-21.

13. Böhler C, Radner H, Ernst M, Binder A, Stamm T, Aletaha $\mathrm{D}$, et al. Rheumatoid arthritis and falls: the influence of disease activity. Rheumatology (Oxford) 2012;51:2051-7.

14. Armstrong C, Swarbrick CM, Pye SR, O'Neill TW. Occurrence and risk factors for falls in rheumatoid arthritis. Ann Rheum Dis 2005;64:1602-4.

15. Hayashibara M, Hagino $H$, Katagiri $H$, Okano $T$, Okada $\mathrm{J}$, Teshima $\mathrm{R}$. Incidence and risk factors of falling in ambulatory patients with rheumatoid arthritis: a prospective 1-year study. Osteoporos Int 2010;21:1825-33.

16. Furuya $\mathrm{T}$, Yamagiwa K, Ikai $\mathrm{T}$, Inoue $\mathrm{E}$, Taniguchi $\mathrm{A}$, Momohara S, et al. Associated factors for falls and fear of falling in Japanese patients with rheumatoid arthritis. Clin Rheumatol 2009;28:1325-30.

17. Stanmore EK, Oldham J, Skelton DA, O'Neill T, Pilling M, Campbell AJ, et al. Risk factors for falls in adults with rheumatoid arthritis: a prospective study. Arthritis Care Res (Hoboken) 2013;65:1251-8.

18. Aydoğ E, Bal A, Aydoğ ST, Cakci A. Evaluation of dynamic postural balance using the Biodex Stability System in rheumatoid arthritis patients. Clin Rheumatol 2006;25:462-7.

19. Duyur Cakit B, Nacir B, Erdem HR, Karagoz A, Saracoglu M. Fear of falling, falls risk and disability in patients with rheumatoid arthritis. Turk J Rheumatol 2011;26:217-25.

20. Smulders E, Schreven C, Weerdesteyn V, van den Hoogen FH, Laan R, Van Lankveld W. Fall incidence and fall risk factors in people with rheumatoid arthritis. Ann Rheum Dis 2009;68:1795-6.

21. Hauer K, Lamb SE, Jorstad EC, Todd C, Becker C. Systematic review of definitions and methods of measuring falls in randomised controlled fall prevention trials. Age Ageing 2006;35:5-10. 